\title{
Biochemical basis of how phosphoserine acidic cluster motifs interact with clathrin adaptors
}

Rajendra Singh ${ }^{1}$, Christopher $\mathrm{Lim}^{2}$, Xiaofei $\mathrm{Jia}^{4}$, Marissa Suarez ${ }^{1}$, Charlotte Stoneham ${ }^{1}$, Yong Xiong ${ }^{2}$, John Guatelli ${ }^{1,3}$

1.Department of Medicine, University of California San Diego (UCSD), La Jolla, California, USA. 2.Department of Molecular Biophysics and Biochemistry, Yale University, New Haven, Connecticut, USA

3. The VA San Diego Healthcare System, San Diego, California, USA.

4. Department of Chemistry and Biochemistry, University of Massachusetts Dartmouth, Dartmouth, Massachusetts, USA

Specific protein-protein interactions are the molecular basis of protein sorting in the endosomal system, a process that controls the trafficking of both host and viral membrane proteins and is highly dependent on clathrin and clathrin adaptors. We aim to understand the molecular and structural basis of how phosphoserine acidic cluster (PSAC) motifs in cellular and viral membrane proteins are recognized by clathrin adaptor protein complexes 1 and 2 (AP-1 and AP-2). AP-1 and AP-2 mediate the trafficking of many transmembrane proteins (cargos) between cellular compartments. Several sorting signals are recognized by AP-1 and AP-2. APcomplexes are heterotetrameric and composed of four subunits. While the medium subunit $(\mu)$ binds to tyrosine-based signals, a hemi-complex of the small $(\sigma)$ and the large, specific subunits binds leucine-based signals. Where PSACs bind on the AP complexes is not clear. Our in vitro binding studies suggest that PSACs interact with basic clusters on the $\mu$ subunits of AP-1 and AP-2. We determined this using both cellular and viral proteins to exemplify these interactions: the cellular proteins Furin and Serinc3 and the viral protein Vpu from HIV-1. All these proteins contain PSAC motifs in their cytoplasmic domains; and specific serines within these PSACs are required for the interaction of these motifs with $\mu$ subunits in a Casein kinase-II- and presumably phosphorylation-dependent manner. Our preliminary in vitro data also suggest that a specific basic patch that is disordered in most crystal structures of $\mu 1$ and $\mu 2$ is part of the target of these PSAC motifs. 